\title{
Ecologia dos aplicativos de mídias sociais da Google Play Store sob a ótica dos métodos digitais e da análise de redes para mídias sociais
}

\author{
Jan Alyne Barbosa Prado' \\ Gabriela da Silva Zago" \\ I - UFOP \\ Ouro Preto (MG), Brasil \\ II - MIDIARS \\ Estados Unidos
}

Resumo: O presente estudo busca experimentar uma combinação de métodos destinados a compreender a configuração da ecologia de aplicativos relacionados às mídias sociais disponíveis na loja Google Play. Partindo de inferências de natureza indutiva e abdutiva ligadas a perspectivas metodológicas e epistemológicas do que Rogers (2014) denomina Métodos Digitais, bem como da Análise de Redes para mídias sociais (RECUERO et al, 2015), a pesquisa busca produzir uma visão mais geral sobre a ecologia dos aplicativos de mídias sociais, a partir das relações de similaridade (processadas por uma ferramenta intitulada Google Play Similar) entre aplicativos estruturadas pela loja Google Play. Os resultados apontam para algumas limitações deste experimento ao mesmo tempo que indicam que a ecologia de aplicativos desta loja se estabelece a partir de uma série de critérios e vetores de atenção para além da similaridade relativa à funcionalidade de tais aplicativos.

Palavras-chave: ecologia dos media; métodos digitais; análise de redes sociais; mídias sociais.

Abstract: Social media app ecology at Google Play Store from the perspective of digital methods and network analysis for social media - This study aims to apply an experiment using a combination of methods, in order to understand the configuration of social media app ecology available on Google Play Store. Starting from inductive and abductive inferences related to methodological and epistemological perspectives that Rogers (2014) calls Digital Methods, as well as the Network Analysis for Social Media (RECUERO et al, 2015), we seek 
to produce a more general view on the ecology of social media apps, considering relations of similarity (processed by a tool called Google Play Similar) between structured applications on Google Play Store. Our results point to some limitations of this experiment, but at the same time indicate that the app ecology is established from a number of criteria and attention vectors beyond the relative similarity to the functionality of such applications.

Keywords: media ecology; digital methods; social network analysis; social media.

\section{Introdução}

Como sistema/ambiente digital complexo, formado por uma estrutura de dados e algoritmos (MANOVICH, 2001), a loja de aplicativos Google Play ${ }^{1}$ (GP) se configura como uma série de sistemas dinâmicos escalares, que processa dados por meio de dispositivos, tais como smartphones ou tablets. Assim como ocorre em qualquer subsistema, podemos observar que não se trata de algo completamente formado, mas de uma ecologia que opera em uma escala diferente (TAFFEL, 2012). Por meio de diferentes escalas de ecologias, torna-se possível observar a emergência de novas qualidades, tendências e capacidades, que não são atribuíveis a qualquer um dos subcomponentes do ecossistema.

A noção de emergência, nesse sentido, propõe que o sistema ecológico como um todo pode apresentar comportamentos que emergem somente e/ou a partir de escalas maiores. A ecologia dos media seria, então, "o estudo das conexões entre atores e processos em sistemas de mídia em várias escalas" (TAFFEL, 2012, online). De modo análogo às relações ecológicas ou interações biológicas de um ecossistema, aplicativos terceiros (third-party apps) se beneficiam de aplicativos que os precedem, sem o prejuízo de seus antecessores, podendo complementá-los, potencializá-los em determinadas funções, redirecioná-los, otimizá-los, entre outros.

Para além dos aplicativos em si, a estrutura de dados do ambiente comporta a quantidade de downloads desses aplicativos, avaliações dos usuários, entre outros, de modo a armazená-los, organizá-los, disponibilizá-los, apresentá-los de modos específicos e recomendá-los a determinados usuários.

Nesse sentido, o presente estudo busca compreender, por meio de inferências de natureza indutiva e abdutiva, a ecologia dos aplicativos de mídias sociais da loja Google Play (GP). Ao identificar os principais aplicativos para cinco ambientes de redes sociais digitais e os aplicativos terceiros recomendados pela GP, podemos compreender os modos pelos quais esses aplicativos estão agrupados por similaridade e são apresentados aos usuários.

Esta investigação é baseada em um projeto pioneiro conduzido com aplicativos voltados para práticas religiosas (DIETER et al, 2015) e realizado por meio de uma combinação de métodos digitais de investigação (ROGERS, 2014), que englobam

1 Disponível em https://play.google.com/store?hl=pt_BR 
ferramentas de extração, tratamento e análise de dados, dentre os quais figura a Análise de Redes Sociais (ARS) (RECUERO et al 2015), de que trataremos adiante. Em síntese, buscamos mapear e compreender a ecologia dos aplicativos ligados a cinco redes sociais digitais: Instagram, Facebook, Twitter, Snapchat e Tinder, no que diz respeito: 1) à configuração do ambiente/espaço no qual os aplicativos da GP estão organizados e relacionados; 2) aos agrupamentos e/ou tipos de ecologia que se formam e se repetem.

\section{Visão geral sobre ecologia dos media}

A ecologia dos media engloba uma série de perspectivas metadisciplinares ${ }^{2}$ relacionadas ao estudo de ambientes de mídia ${ }^{3}$, ou sistemas de comunicação complexos. Ecologia, para Strate (2002) implica o estudo dos ambientes: sua estrutura, conteúdo e impacto sobre as pessoas. Essa noção se estrutura a partir da ideia de que distintas práticas culturais são configuradas conforme a apropriação de tecnologia, técnicas, sistemas de informação e códigos de comunicação, a partir dos quais emergem diferentes escalas.

Tais escalas produzem camadas e interoperam em ambientes complexos de mídia, e como cada escala vem com e criou suas próprias dimensões relacionais: política, material, estética, e outras dinâmicas, que a geraram e a partir das quais podem ser lidas. (...) Uma infinidade de outras forças de composição e domínios que o colocam em relação a outros que podem ser virtuais, afetivas, históricas, e assim por diante (FULLER, 2005, p.187)

Algumas noções ligadas à ecologia dos media sistematizadas por Postman (apud STRATE, 2002) sobre ambiente são úteis para pensarmos o nosso objeto de estudo, a saber, a ecologia dos aplicativos para mídias sociais disponíveis na GP. Segundo o autor, ecologia dos media pode ser definida como o estudo dos media como ambientes, um sistema complexo de mensagens ou códigos que estabelecem aos seres humanos certas maneiras de pensar, sentir e agir. Especifica e explicita condições para gramáticas de performance, de modo a estruturar o que é possível ver, dizer e/ou fazer, e a designar atribuições para que possamos desempenhá-las, configurando ainda possibilidades e limites para ação e definição de papéis.

Altheide (1994, p. 668, tradução nossa) ressalta a importância da ecologia da comunicação, como algo que se dá a partir das relações estabelecidas em ambientes de mídia, no sentido de "nos tornarmos mais atentos a essa interação que envolve tecnologia

2 Strate (1999, online, tradução nossa) argumenta que "a emergência de novos campos de investigação é tão ampla em seu escopo que a palavra 'disciplina' sugere a existência de uma área de especialização muito bem delimitada", o que pouco se aplicaria aos estudos de ecologia dos media.

3 Alt (2011) traça um panorama histórico com vistas a entender como a Programação Orientada a Objetos (POO) modifica o status da cultura do computador, para além da noção de medium como um sistema de armazenamento. A POO atualizaria, portanto, a condição do computador como configurador de sistemas/ ambientes digitais complexos. 
da informação, formatos e atividades, de modo a reconhecer processos sociais significativos que estão transformando nossas vidas".

Citton (2017), por sua vez, defende que os media devem ser observados mais como um ecossistema, em oposição à ideia de "canal" de transmissão:

Este ecossistema funciona como uma câmara de eco, cujas reverberações 'ocupam' nossas mentes (no sentido militar do termo): na maioria das vezes, pensamos (...) apenas o que faz com que a caixa forte dos media ressoe em nós pelos ecos que nos rodeiam. Em outras palavras, os mecanismos dos media que capturam nossa atenção e nos despertam fascínio criam um ecossistema, entendido como uma infraestrutura de ressonâncias que condiciona nossas atenções ao que circula através e dentro de nós (CITTON, 2017, p. 29, tradução nossa).

Ao propor uma ecologia da atenção, e considerando a complexidade que permeia as interações que envolvem sujeitos, media, objetos etc., Citton (ibidem, p. 34) discorre sobre a coletivização da atenção, no sentido de que esta prescinde da coleta de características comuns que nos ajudam a prosperar em nosso entorno, uma vez que a atenção seletiva "serve (...) para filtrar fenômenos do (...) meio ambiente e para constituir comunidades de sensibilidade e ação". A atenção seletiva "assegura simultaneamente uma certa adaptação do nosso comportamento ao (...) meio ambiente (selecionando o que nos interessa) e uma certa composição coletiva dos desejos individuais (alinhando [...] nossas sensibilidades e [...] preferências com as dos outros)".

Esta composição coletiva é movida pelo que o autor denomina "Poder Vetorialista", que consiste no "poder de mover e combinar tudo e qualquer coisa como um recurso", e cuja classe é "composta por todos aqueles que controlam e lucram com a vetorialização material necessária da informação, (...) através do controle dos vetores ao longo dos quais o financiamento dos investimentos que irrigam [as] empresas atravessa" (ibidem, p. 63).

No caso dos ambientes digitais, observa-se o desenvolvimento de novos vetores de atenção, cognição e ação, que "induzem efeitos quantitativos que alteram qualitativamente a orientação de nossa atenção digitalizada" (ibidem, p. 66). Os agrupamentos dos aplicativos da GP são impulsionados, portanto, por alguns desses vetores, uma vez que prescindem de critérios de padronização e hierarquização ${ }^{4}$, configurados a partir de procedimentos de programação, isto é:

(...) num protocolo que, ao determinar a entrada do continuum concreto como dados abstratos, materialmente (e não apenas culturalmente), preconfigura nossa percepção da realidade. (...) A digitalização de nossa atenção submete aos efeitos de programação inerentes aos vetores, que permitem que ele circule mais rapidamente, mais amplamente e mais intensamente do que nunca (ibidem, p. 68).

4 Para conhecer mais sobre tais critérios, ver Citton, 2014. 
Tais considerações coadunam com os princípios de comodificação e de quantificação: o primeiro explica a agregação maquínica da atenção coletiva por meio do desenvolvimento de fluxos de atenção que visam a atender às necessidades e aos desejos que maximizem os retornos financeiros. Já o segundo corresponde à agregação mecânica da atenção, que "produz uma figura que atribui um valor de atenção a cada entidade ao mesmo tempo t. Estamos realmente lidando aqui com uma taylorização da mente: a magia do Google baseia-se na automação do processo coletivo através do qual atribuímos valor às coisas que compõem nosso mundo" (ibidem, p. 74).

Embora seja impossível acessar os protocolos que impulsionam todos os vetores de atenção na GP, é possível coletar algumas pistas que estruturam o campo de visibilidade dos apps de mídias sociais e, mais especificamente, a forma como eles estão agregados, por meio de um protocolo de investigação construído pela Digital Methods Initiative ${ }^{5}$, do qual trataremos em seguida.

\section{Métodos Digitais}

Métodos digitais, em síntese, dizem respeito ao desenvolvimento de novas perspectivas epistemológicas, metodológicas e de uma mentalidade direcionada à pesquisa social com a Web.

O objetivo dos métodos digitais, para Rogers (2014), é reorientar o domínio da investigação na internet, ao estudar e redefinir o que ele chama de métodos do medium, na medida em que evoluem, a saber: métodos incorporados aos dispositivos online que abrangem as técnicas para coleta, classificação e análise de dados nativamente digitais (a exemplo de tags, hiperlinks, etc.), visando à produção de diagnósticos culturais e sociais (ibidem).

Langlois (2014) segue a perspectiva postulada por Rogers (2014), quando discute o papel dos softwares como máquinas (semi) automáticas de produção de sentido. Tal concepção é útil para pensar que tais dispositivos potencializam o agenciamento da produção de conhecimento, em diversas instâncias, sejam estas relativas não somente à circulação de dados em ambientes digitais, como também no que diz respeito à coleta, ao tratamento e à análise de dados nativamente digitais.

Segundo Rogers (ibidem), os métodos digitais não buscam necessariamente ancorar (todas) as descobertas nos ambientes digitais, mas colocar a questão do status da Web como ancoragem em potencial para se pensar fenômenos, comportamentos e práticas sociais e culturais.

Dieter et al (2015) realizaram um estudo com os aplicativos da GP, buscando descobrir como essa loja organizava o espaço da eecologia de aplicativos ligados a práticas religiosas. De modo similar, no presente estudo, buscamos compreender a ecologia dos aplicativos

5 https://www.digitalmethods.net 
de mídia social na GP. Inicialmente, procedemos à coleta de dados, constituídos pelos primeiros 100 aplicativos extraídos da base de dados da GP, obtidos através do diretório da empresa de serviços de análise e inteligência para o mercado App Annie ${ }^{6}$, de acordo com as seguintes palavras-chave: "Facebook", "Twitter", "Instagram", "Snapchat" e "Tinder".

Após extrair a identidade dos 100 aplicativos relacionados a cada uma das mídias sociais listadas acima, extraídas do App Annie por meio da ferramenta Harvester ${ }^{7}$ e de um editor de texto, inserimos as informações obtidas na ferramenta Google Play Similar ${ }^{8}$, que gera um arquivo em gexf com aplicativos similares aos 100 aplicativos iniciais, para então proceder à Analise de Redes com o uso do software Gephi. Os grafos foram gerados a partir da utilização do layout ForceAtlas2, que tem por objetivo aproximar nós que apresentam conexões em comum.

As principais métricas consideradas foram modularidade, grau do nó e grau de intermediação. A primeira delas "identifica os grupos subjacentes à estrutura da rede", separada por módulos ou comunidades, isto é, "grupos de nós densamente interligados entre si e fragilmente conectados com o resto da rede" (RECUERO et al, 2015, p. 84). Nesse sentido, a modularidade foi utilizada para identificar os clusters que compõem a rede, podendo revelar "características estruturais inesperadas" (ibidem, p. 85). O grau de modularidade vai de 0 a 1 . Redes com alta modularidade (ou seja, com grau mais próximo a 1) tendem a ter conexões densas entre os nós de cada grupo e poucas conexões com nós de outros grupos.

O grau do nó "representa o número de conexões que um determinado nó possui" (ibidem, p.66), podendo se desdobrar em grau de entrada (indegree), que representa as conexões recebidas, e grau de saída (outdegree), que representa as conexões enviadas. O grau do nó foi utilizado para identificar os aplicativos centrais da rede. Já o grau de intermediação, por sua vez, é uma métrica que "mostra quais nós [no caso, aplicativos] são mais relevantes para conectar diferentes grupos" (ibidem, p. 69). Em outras palavras, essa métrica foi utilizada para identificar os aplicativos que servem de ponte para conectar pontos distantes da rede de cada aplicativo.

Na análise realizada, cada app foi considerado um "nó" da rede, ao passo que o fato de aparecer como similar a outro app da mesma rede foi considerado como "conexão". Assim, cada rede parte de 100 nós originais (os 100 resultados da busca) e suas relações de similaridade com outros apps. Para cada aplicativo exibido, a GP sugere até 24 apps "similares".

A análise de redes sociais foi utilizada não somente para identificar a estrutura das redes em torno dos aplicativos, como também para observar a ecologia que se estrutura a partir deles, uma vez que executam determinadas funções relacionadas a apropriações

6 https://www.appannie.com/

7 https://tools.digitalmethods.net/beta/harvestUrls/

8 https://tools.digitalmethods.net/beta/googlePlaySimilar/ 
e usos ligados às mídias sociais. A estrutura de rede formada em torno dos aplicativos de mídias sociais nos mostra, portanto, um tipo de tecnicidade circundante às performances associadas aos seus usos e apropriações. Ainda que a análise de redes sociais seja utilizada fundamentalmente para a análise de estruturas sociais (ou seja, entre pessoas), consideramos pertinente apropriá-la para o estudo da ecologia dos media, considerando seu poder vetorialista, na acepção de Citton (2017). Em outras palavras, consideramos as relações entre os nós como unidade de análise, ou seja, no caso específico deste artigo, o foco recai sobre vetores que agregam relações de similaridade entre os apps, e não necessariamente nos apps em si. Além disso, a ecologia dos media consiste em um pano de fundo teórico para compreender a ecologia dos apps, porém não indica caminhos metodológicos precisos. Disso decorre a necessidade de combinar essa perspectiva teórico-metodológica com os procedimentos e métricas da ARS.

A metáfora da ecologia, no caso desta pesquisa, se baseia no estudo sobre as "condições de existência dos seres vivos e as interações, de qualquer natureza, existentes entre esses seres vivos e seu meio" (CASSINI, 2005). Uma vez que a ARS se refere à descrição das relações entre atores (aplicativos) e suas relações configuradas a partir do Google Play Similar, defende-se que é possível, nesse sentido, observar dados relacionais estruturados a partir dessas conexões e desses vetores de atenção, de modo a propor uma tradução para a metáfora de ecologia, considerando os objetos digitais, a saber, os aplicativos organizados e disponibilizados na loja GP.

Para Van Dijck (2013, p.11, tradução nossa), "a própria palavra 'social' associada a 'mídia' implica que as plataformas são centradas no usuário e facilitam atividades coletivas, da mesma forma que o termo 'participativo' enfatiza a colaboração humana. De fato, as mídias sociais podem ser vistas como facilitadoras ou aprimoradoras das redes humanas". Aplicativos de mídias sociais, nesse sentido, são aplicativos para dispositivos móveis que permitem o acesso, a atualização e a manutenção de redes sociais na internet.

A escolha dos aplicativos se deu por razões ligadas à popularidade, a funcionalidades e ao caráter de novidade. Em termos de popularidade, três - Facebook, Twitter e Instagram - figuram entre as mídias sociais mais utilizadas no Brasil ${ }^{9}$. Com relação às funcionalidades, também se buscou exemplo do uso específico para paquera e namoro, como é o caso do Tinder. A novidade, no caso do Snapchat, foi outro critério, no sentido de compreender que tipos de apps terceiros se assemelham a apps emergentes.

\section{Resultados}

A Tabela 1 traz uma síntese dos dados obtidos com a análise das redes em torno dos aplicativos Facebook, Twitter, Instagram, Snapchat e Tinder.

9 O ranking das mídias/redes sociais mais usadas no Brasil conforme resultados da Pesquisa Brasileira de Mídia 2015 pode ser consultado em: http://bit.ly/1FAvjZC 


\begin{tabular}{|l|l|l|l|l|}
\hline Rede & Nós & Conexões & Número de módulos & Modularidade da rede \\
\hline Facebook & 742 & 1.455 & 46 & 0.706 \\
\hline Twitter & 382 & 1.702 & 26 & 0.363 \\
\hline Instagram & 641 & 1.917 & 23 & 0.693 \\
\hline Snapchat & 322 & 710 & 23 & 0.653 \\
\hline Tinder & 258 & 511 & 17 & 0.716 \\
\hline
\end{tabular}

Tab. 1. Síntese dos dados obtidos. Fonte: métricas calculadas com a utilização do software Gephi 0.8.1 beta. Modularidade calculada através do algoritmo de Blondel et al (2008).

A seguir são apresentados os resultados obtidos na análise estrutural das relações em torno dos aplicativos similares a cada uma das redes analisadas.

\section{Facebook}

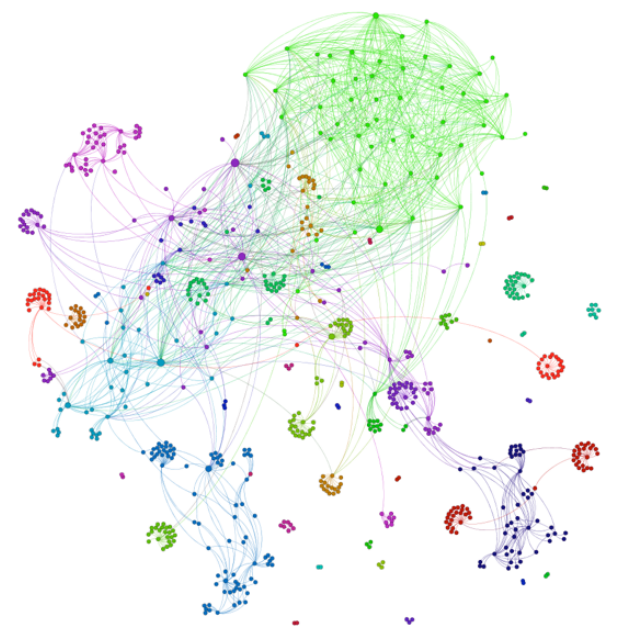

Fig. 1. Aplicativos similares ao Facebook na Google Play.

Nesta rede, foi identificado um total de 46 módulos ou grupos, constituindo clusters em torno de determinados aplicativos. São ao todo 742 aplicativos diferentes e 1.455 relações de similaridade entre eles. Essa rede tem uma composição peculiar na medida em que possui poucos módulos maiores e vários menores, centrados em aplicativos para propósitos específicos.

O maior grupo da rede se encontra na parte inferior esquerda do grafo, na cor azul (em tom médio), e abrange ferramentas diversas para Facebook, incluindo downloaders (de fotos e vídeos) e acessórios. No total, são 85 aplicativos. O segundo maior grupo da rede está localizado na parte superior esquerda do grafo, na cor roxa, e encontra-se parcialmente misturado a outros módulos. São 63 aplicativos, a maioria composta por editores de foto, não necessariamente relacionados diretamente ao Facebook. 
O grupo verde, na parte superior central do grafo, é o terceiro maior módulo, com 56 aplicativos, composto por diversas ferramentas para Facebook, quase todas com o termo "Facebook" no nome. A seguir, há um grupo na cor azul marinho, na parte inferior direita do grafo, com 49 aplicativos. Esse grupo abrange aplicativos que fornecem conteúdo para o Facebook (frases, stickers e fotos). Vários desses aplicativos são em outros idiomas que não o inglês. O grupo 41, na cor laranja, com 46 apps, à esquerda do grafo, inclui aplicativos de atualização de status e com frases, sendo que uma parte deles é voltada para Whatsapp e não Facebook. O Facebook, seus aplicativos oficiais (Groups, Messenger, e outros), e outras redes sociais similares (Twitter, Skype, Hangouts e outros) se encontram no grupo de cor azul piscina, do lado esquerdo ao centro do grafo. O módulo verde, à esquerda do grafo, inclui 23 apps de notificações para smartwatch.

Os dois apps com maior grau de intermediação são voltados para socialização: SimpleChat PRO for Facebook, que permite acessar o chat do Facebook, e Facebook Groups, app oficial do Facebook para acessar e administrar grupos. O terceiro app com maior grau de intermediação, entretanto, está no grupo roxo que atravessa os principais grupos do grafo, indicando que possui relações de similaridade com apps de vários outros grupos.

Dentre os apps com maior outdegree, ou seja, aqueles que mais vezes apareceram relacionados como similares a outros apps da rede, é possível identificar diferentes apps de terceiros que oferecem complementos ao Facebook (como Fast Pro for Facebook, ou Puffin for Facebook). Também é possível observar apps que possuem o Facebook como desenvolvedor, como nos casos do Free Basics by Facebook e do Facebook Groups.

\section{Twitter}

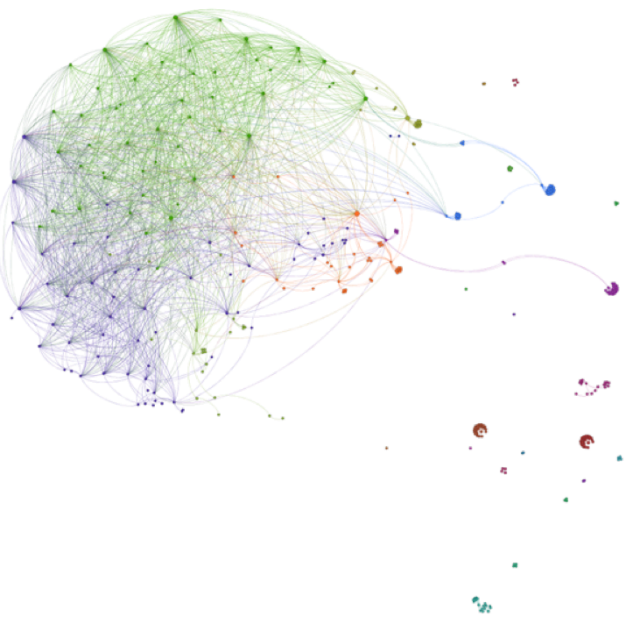

Fig. 2. Aplicativos similares ao Twitter na Google Play. 
Nesta rede, foram identificados, no total, 26 grupos. São ao todo 382 nós e 1.702 conexões. A modularidade dessa rede é relativamente mais baixa que a das demais (vide Tabela 1), o que em termos práticos significa que há muitas conexões entre os grupos.

Os dois maiores clusters da rede estão localizados à esquerda do grafo, bastante próximos um do outro. No grupo roxo, a maior parte dos aplicativos inclui clientes de Twitter, e alguns aplicativos em japonês. O grupo verde inclui tanto clientes quanto gerenciadores de Twitter. Esses dois grupos estão bem próximos entre si justamente por conter apps com propósitos parecidos e com conexões em comum.

O terceiro maior cluster da rede está localizado entre os dois grupos anteriores (verde e roxo). Esse grupo tem uma característica um pouco peculiar por conter muitos aplicativos relacionados ao Instagram (como gerenciadores de perfis, ferramentas para repostagem, dentre outros), em especial aplicativos para conseguir mais seguidores. O grupo também inclui alguns apps de Twitter para propósitos similares (em especial gerenciadores de perfis e aplicativos para conseguir seguidores), o que ajuda a explicar a proximidade física com os outros dois grupos.

Alguns grupos peculiares aparecem em pontos mais afastados da rede. Como exemplo, o grupo marrom está desconectado do resto da rede e inclui apps relacionados à leitura (livros, audiobooks, leitores de PDF, etc.). O aplicativo Twitter Tricks (livro de dicas sobre o Twitter) é o único sobre Twitter nesse grupo, e provavelmente é o aplicativo que fez com que os demais aplicativos fossem incluídos na amostra, por serem relacionados a ele.

Já o grupo lilás está interconectado ao resto da rede por alguns aplicativos-ponte, mas no geral inclui aplicativos de música. A ponte com o grupo principal é estabelecida por um conjunto de aplicativos da Sony com extensões para celulares da marca. O conjunto de extensões inclui um app de Twitter (que está junto aos demais aplicativos no grupo principal) e duas extensões de música (que estão no meio do caminho, conectando o grupo principal ao grupo isolado).

Na rede em torno do Twitter, os apps com maior grau de intermediação estão principalmente no grupo verde, e incluem ferramentas e gerenciadores de Twitter (como Unfollowers e TwitGrow). O próprio Twitter também exerce o papel de interconectar a rede. Já em termos de maior outdegree predominam apps de clientes de Twitter, o que traz indícios de que os clientes aparecem bastante como similares a outros apps relacionados ao Twitter, ainda que não necessariamente contribuam para interconectar a rede. 


\section{Instagram}

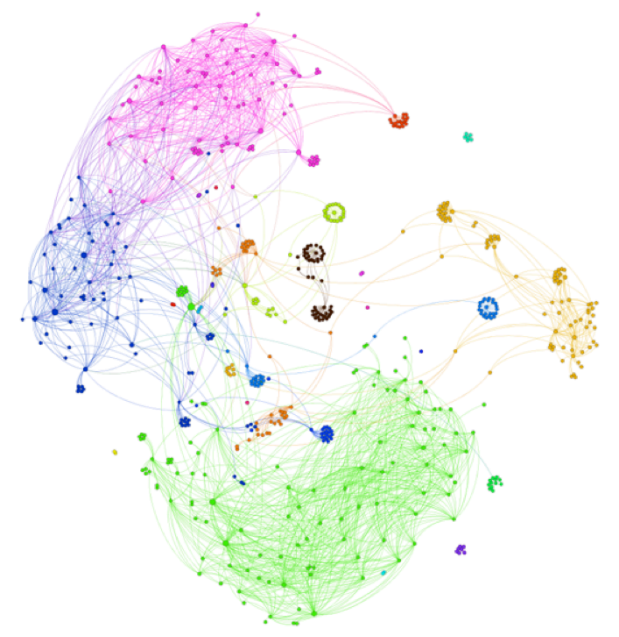

Fig. 3. Aplicativos similares ao Instagram na Google Play.

Na rede em torno do Instagram, foram identificados 23 módulos. O maior deles, em verde claro na parte inferior do grafo, engloba ferramentas para Instagram (para conseguir seguidores, e também para repostagem, hashtags, e outros). Neste grupo também estão outras grandes redes sociais (como Facebook e Twitter). À direita do grafo, há um módulo amarelo que também conta com ferramentas para Instagram, porém mais voltadas para vídeo e/ou download de fotos. Este grupo também apresenta alguns aplicativos para smartwatch.

Na parte superior do grafo, há dois grandes grupos: um rosa e um azul, que trazem ferramentas para Instagram, com foco especial em editores de foto para postar na ferramenta. No centro do grafo há um grupo marrom, que traz ferramentas para gerar wallpaper/imagem para a tela bloqueada a partir do Instagram, bem como ferramentas para criar mensagens de texto para postar no Instagram.

Dentre os apps com maior grau de intermediação, estão o próprio Instagram (com o maior grau) e ferramentas voltadas para Instagram, em especial para edição de fotos (como RetroShots + voor Instagram, ou No Crop Pic voor instagram). Também há alguns outros acessórios de Instagram não necessariamente voltados para fotografia, como Crowdfire for Instagram growth, voltado para administrar seguidores e agendar posts no Instagram. Já na lista de apps com maior outdegree, predominam ferramentas relacionadas a seguidores de Instagram e não editores de foto. Assim, quantitativamente, esse tipo de app apareceu mais vezes como similar a outros apps da rede, ainda que apps de foto tenham contribuído em maior intensidade para interconectar a rede. Apps voltados para sugestões de tags para Instagram também aparecem na relação de apps com maior outdegree. 


\section{Snapchat}

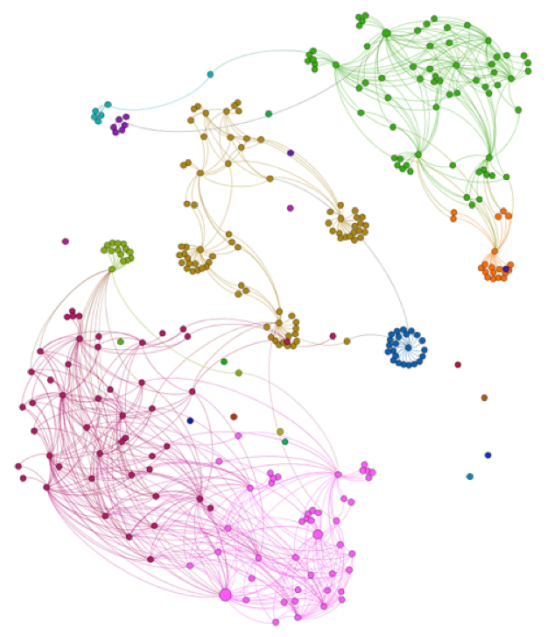

Fig. 4. Aplicativos similares ao Snapchat na Google Play.

Identificamos um total de 23 módulos na rede em torno do aplicativo Snapchat. O módulo amarelo traz aplicativos com emojis e guias para uso do Snapchat. O verde, à direita do grafo, traz acessórios de vídeo e foto. O módulo rosa escuro, por sua vez, traz aplicativos diversos para Snapchat. Já o grupo ao lado, em rosa em tom mais claro, é onde estão o Snapchat e outros aplicativos gerais de redes sociais (como Facebook, Twitter e outros).

O módulo azul no meio do grafo traz vários aplicativos de segurança (para bloqueio de apps) e está conectado ao grupo amarelo, com guias para uso do Snapchat. O módulo laranja traz editores de foto/montagem (mais voltados para Instagram). Esse módulo está próximo ao grupo verde, que traz também acessórios de foto e vídeo.

Na rede em torno do Snapchat, apenas três apps possuem grau de intermediação considerável, exercendo papel de interconectar a rede: o próprio Snapchat, o aplicativo DashClock Snapchat Extension, e o aplicativo Snappic - Photo Editor. O DashClock permite visualizar notificações do Snapchat na tela bloqueada. Já o Snappic não possui relação direta com o Snapchat - o propósito do aplicativo é salvar fotos facilmente, do mesmo modo como fotos são salvas no Snapchat. Esse aplicativo está no grupo verde, à direita do grafo, e exerce o papel de interconectar os grupos de aplicativos associados a fotografia e imagem (na medida em que também aparece como similar a apps do grupo laranja).

A situação é um pouco diferente ao se observar os apps com maior outdegree, ou seja, aqueles que mais apareceram como similares a outros apps da rede. Nessa relação aparecem ferramentas acessórias ao Snapchat e a outros apps similares (o app com maior 
outdegree, por exemplo, chama-se Grab for Instagram, uma ferramenta para Instagram que possui integração com outras redes sociais, incluindo Snapchat).

\section{Tinder}

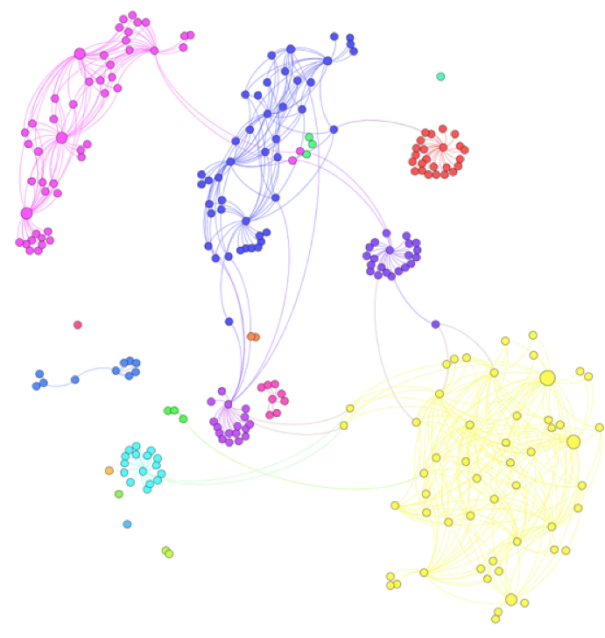

Fig. 5. Aplicativos similares ao Tinder na Google Play.

A rede do Tinder possui 17 módulos. O maior módulo, em amarelo, traz muitos apps voltados para "descobrir" coisas - como comida, ofertas, etc. Inclui também outros aplicativos de namoro, entre eles alguns voltados para grupos específicos (judeus, indianos, asiáticos, etc.). O interessante nesse grupo é que traz aplicativos similares ao Tinder em duas frentes diferentes: apps voltados para descoberta de coisas novas e apps voltados para conhecer pessoas novas. O rosa pink, na parte superior do grafo, encontra-se bastante afastado do módulo amarelo. Ele inclui aplicativos de namoro similares ao Tinder e alguns acessórios de Tinder. O Snapchat está presente nesse grupo. O módulo azul também traz vários acessórios para Tinder (para esconder localização, frases, editor de foto, etc.).

O módulo laranja traz aplicativos de música em geral. Um dos apps nesse grupo chama-se Tinderbox Festival e está relacionado a isqueiro (tinder-box). O módulo roxo é onde estão o Tinder e alguns aplicativos similares, como Skype, Whatsapp, e outros mensageiros.

O grupo lilás traz aplicativos de fotos - incluindo um chamado Wild Selfie for Tinder. Os aplicativos com maior grau de intermediação do Tinder estão nos três maiores grupos e exercem papel de interconectar a rede. Compreendem, em geral, ferramentas voltadas para Tinder (Dating tips Guide for Tinder e pick-up lines tinder, com dicas e frases para usar no Tinder, ou Swipely for Tinder, voltado para facilitar a tarefa de curtir ou passar fotos no Tinder). Também há, nesse grupo, a presença do FoodMe - It's Tinder for Food, que seria um app similar ao Tinder, porém voltado para comida. 
Dentre os apps com maior outdegree, há tanto apps parecidos com os propósitos do Tinder, como ferramentas para Tinder (como Swipely for Tinder ou Dating Tips Guide for Tinder, que também possuem alto grau de intermediação). O app com maior outdegree é GetHomeSafe, cujo propósito é garantir a segurança de indivíduos que estejam se deslocando a determinados lugares. Na descrição do app consta que ele pode ser usado como medida de segurança quando se vai a encontros marcados por meio do Tinder (além de inúmeras outras aplicações possíveis).

\section{Considerações finais}

O trabalho procurou identificar as relações de similaridade a partir de cinco aplicativos de mídias sociais disponibilizados na Google Play Store. Essas relações foram observadas por meio de um protocolo dos métodos digitais, em especial a partir das métricas de análise de redes sociais: modularidade, grau do nó e grau de intermediação.

Algumas limitações do estudo decorrem do próprio modo como a ferramenta utilizada para coleta de dados se estrutura. Ao navegar na GP, um total de até 24 aplicativos relacionados é exibido. Assim, a ferramenta Google Play Similar, utilizada para a coleta de dados do presente trabalho lista até 24 apps como similares a cada um dos 100 apps coletados, o que significa que esses 100 apps iniciais possuem indegree entre 1 e 24 (conforme o número de apps similares que possuem). Todos os demais apps captados como similares a esses apps recebem indegree 0 (pois não foi olhada a rede deles). O outdegree, por sua vez, é calculado conforme o número de vezes que cada app aparece como similar a outros apps.

No caso deste experimento, em que os aplicativos foram coletados separadamente no diretório App Annie a partir das palavras-chaves ligadas às mídias sociais selecionadas como objeto da pesquisa, a métrica que teria potencialmente maior relevância seria o outdegree, uma vez que indica quantas vezes cada app aparece como similar a outros apps da rede. A partir desse resultado é possível observar as estruturas de similaridade estabelecidas pelo algoritmo.

Em síntese, podemos concluir que alguns vetores que indicam relações de "similaridade", estruturadas pelo Google Play Similar, conformam ecologias de apps de mídias sociais, cuja tecnicidade se estabelece: 1) mais pelas propriedades relativas às funcionalidades do que pelos apps em si. Exemplos disso são apps para conseguir mais seguidores, ou para fazer repostagem, que podem servir tanto para o Twitter quanto para o Instagram, a título de exemplo; 2) a partir de funcionalidades para aplicativos específicos (como é o caso dos clientes e gerenciadores para Twitter ou chats incorporados ao Facebook, editores de fotos para o Instagram e demais aplicativos de mídias sociais); 3) por conexões entre aplicativos pertencentes a uma mesma empresa (o caso do Whatsapp e do Facebook), e não pelas funções que desempenham; 4) por aplicativos desenvolvidos para dispositivos específicos (a exemplo dos celulares da Sony e de apps para smartwatch). 
As ecologias encontradas trazem indícios de como se organizam os apps de mídias sociais na GP. Compreender as ecologias que se formam em torno de cada app permite compreender como os apps se estruturam na loja, em termos de vetores que estruturam algumas relações de similaridade propostas pela ferramenta. Isso traz implicações ao usuário, na medida em que os algoritmos de similaridade sugerem aplicativos suspostamente relacionados àqueles visitados na GP. Compreender como essas relações se estabelecem auxilia a desvendar alguns vetores de atenção que perpassam as ecologias em torno dos aplicativos para mídias sociais.

Embora a ecologia dos apps de mídias sociais operacionalizada neste estudo evidencie certos aspectos ligados a algumas práticas levadas a cabo em mídias/redes sociais digitais, a saber, a similaridade de ferramentas que conformam uma tecnicidade destinada a facilitar as conexões, ou ainda a produção/edição/circulação de conteúdos nessas redes, o agrupamento dos apps impossibilita a observação da similaridade segundo outras métricas. E, nesse sentido, fazem-se necessários outros experimentos, em que seja possível analisar relações de similaridade considerando os aplicativos de mídias sociais em conjunto, de modo a observar as redes que se constituem em torno deles.

Este experimento também busca estimular o desenvolvimento de métodos digitais de pesquisa, de forma a traduzir a ecologia dos apps de modo mais amplo, considerando diferentes escalas, com vistas a observar a emergência de novas qualidades, tendências e capacidades desse subsistema. A título de exemplo, como se estabelece a organização do espaço dos aplicativos de mídias sociais estabelecido pela loja de aplicativos da GP em detrimento da experiência de navegação do usuário? Ele segue a lógica estabelecida pela loja ou resiste a ela? Como esses espaços são organizados ou agrupados em torno de outras práticas, tais como o meio ambiente, os esportes, entre outros? O que a organização desse espaço pode dizer sobre o lugar da cultura no que diz respeito ao uso dos apps de mídias sociais e sua relação com outras práticas? Tal experimento se configura como uma ponta de lança para se pensar tais questões.

Jan Alyne Barbosa Prado é professora de Comunicação Social da Universidade Federal de Ouro Preto (UFOP) e doutora em Comunicação e Cultura Contemporâneas pela Universidade Federal da Bahia (UFBA).

janalyne@gmail.com

Gabriela da Silva Zago é pesquisadora visitante da University of Oregon e integrante do Grupo de Pesquisa em Mídia, Discurso e Análise de Redes Sociais (MIDIARS). É doutora em Comunicação e Informação pela Universidade Federal do Rio Grande do Sul (UFRGS).

gabrielaz@gmail.com 


\section{Referências}

ALT, C. Objects of Our Affection. How Object Orientation Made Computers a Medium. In: PARIKKA, J.; HUHTAMO, E. In: Media Archaeology. California: University of California Press, 2011.

BLONDEL, V. et al. Fast unfolding of communities in large networks. 25 Jul. 2008. Disponível em: https://arxiv.org/pdf/0803.0476.pdf. Acesso em: 24 Jan.2018.

CASSINI, S. T. Ecologia: Conceitos Fundamentais. 2005. Disponível em http://bit.ly/2tglzTg Acesso em: 08 Mar. 2016.

CITTON,Y. The Ecology of Attention. Kindle Edition. Malden: Polity Press, 2017.

DIETER et al. Digital Methods for App Analysis: Mapping App Ecologies in the Google Play Store, 2015. Disponível em http://bit.ly/1KeWA4V. Acesso em 05 Mar.2017

FULLER, M. Media Ecologies. New York: MIT Press, Kindle Edition. 2005

LANGLOIS, G. Meaning at the age of social media. New York: Palgrave Macmillan, 2014.

MANOVICH, L. The language of new media. Cambridge: MIT Press, 2001.

RECUERO, R. et al. Análise de redes para mídia social. Porto Alegre: Ed.Sulina, 2015.

ROGERS, R. Digital Methods. Cambridge: MIT Press, 2014.

STRATE, L. Media Ecology as a Scholarly Activity. Proceedings of the Media Ecology Association, v.3, 2002.

TAFFEL, S. Media Ecology. An Introduction. Disponível em https://mediaecologies.wordpress.com/ media-ecology-an-introduction/ Acesso em: 06 Out. 2012.

VAN DIJCK, J. The Culture of Connectivity. New York: Oxford University Press, 2013.

Artigo recebido em 30 de abril e aprovado em 06 de julho de 2017. 